\title{
Prediction of minimal encephalopathy in patients with HCV-related cirrhosis using albumin-bilirubin, platelets-albumin- bilirubin score, albumin-bilirubin-platelets grade and ammonia level
}

\author{
Ayman Alsebaey
}

\begin{abstract}
Background: Minimal hepatic encephalopathy (MHE) is a complication of liver cirrhosis causing low quality of life, driving skills and higher traffic violation. The neuro-psychometric tests are the gold standard but difficult clinically and time-consuming. The aim was to assess albumin-bilirubin (ALBI), platelets-albumin-bilirubin (PALBI) score, albumin-bilirubin-platelets (ALBI-PLT) grade and ammonia level as MHE predictors. All the patients $(n=257)$ underwent critical flicker frequency number connection, serial dotting and digit symbol test for MHE diagnosis ( $n=166,64.6 \%$ ). Liver function, INR, CBC and arterial ammonia were measured.
\end{abstract}

Results: There was statistically significant difference $(p<0.05)$ between MHE patients and those without as regards ammonia ( $86.59 \pm 23.25$ vs. $63.56 \pm 24.2 \mu \mathrm{mol} / \mathrm{L})$, ALBI score $(-2.13 \pm 0.53$ vs. $-2.49 \pm 0.38), \mathrm{PALBI}$ score $(-2.33 \pm 0.39$ vs. $-2.55 \pm 0.26)$ and ALBI-PLT (3.98 \pm 0.49 vs. $3.70 \pm 0.56)$. Patients with MHE were mainly Child-Pugh B and C and also ALBI grade 2 and 3. For MHE discrimination, ALBI, PALBI, ALBI-PLT and ammonia had the following cutoffs $>-2.36$ (57.23\% sensitivity, $77.78 \%$ specificity), $>-2.5$ (60.84\% sensitivity, $67.9 \%$ specificity), $>3$ ( $87.35 \%$ sensitivity, $27.16 \%$ specificity) and $>76.5$ (69\% sensitivity, $72.5 \%$ specificity) respectively $(p=0.001)$. On comparison of the area under the curve, ALBI is comparable to PALBI ( $p=0.245)$ and ammonia $(p=0.603)$. The ALBI-PLT is inferior to ALBI $(p=0.018)$ and ammonia $(p=0.021)$ but comparable to PALBI $(p=0.281$ ). ALBI (odds = 5.64), PALBI (odds = 7.86), ALBI-PLT (odds = 2.86), ammonia (odds = 1.05), Child-Pugh score (odds $=2.13$ ), MELD (odds $=1.26$ ) are independent predictors of MHE.

Conclusion: ALBI, PALBI and ammonia are clinical useful model for MHE prediction.

Keywords: Minimal hepatic encephalopathy, Cirrhosis, ALBI, PALBI, Ammonia

\section{Background}

Hepatic encephalopathy is simply brain dysfunction owing to acute or chronic liver disease. It is of two types; overt and covert type. Overt type is characterized by bedside characteristic clinical features and does not need sophisticated investigations for diagnosis [1].

Covert or minimal hepatic encephalopathy (MHE) is characterized by an examination by normal mental and

\footnotetext{
Correspondence: aymanalsebaey@liver.menofia.edu.eg Department of Hepatology and Gastroenterology, National Liver Institute, Menoufia University, Shebeen Elkoom 32511, Egypt
}

neurological status. It can be diagnosed by sophisticated psychometric tests, e.g., paper-and-pencil psychometric tests, inhibitory control test, critical flicker frequency and the stroop smartphone application [2, 3].

Up to $80 \%$ of patients with cirrhosis have MHE. Its presence is associated with poor quality of life, inability to drive, traffic violation and accidents. Within 3 years, $\sim 50 \%$ may develop overt hepatic encephalopathy $[1,4]$.

MHE is commonly found with advanced liver disease, history of overt hepatic encephalopathy, esophageal varices and alcohol abuse as etiology of liver cirrhosis [5]. 
Since the MHE investigations are expensive, cumbersome and time-consuming, it is important to select the patients that need to undergo them with high yield.

This study aimed to assess albumin-bilirubin (ALBI), platelets-albumin-bilirubin (PALBI) score, albuminbilirubin-platelets (ALBI-PLT) grade and ammonia level as noninvasive predictors of MHE.

\section{Methods}

This study was conducted in National Liver Institute Hospitals, Menoufia University, Egypt. After institutional review board approval, an informed consent was obtained before inclusion in the study.

Our study included 257 patients diagnosed to have HCV-related liver cirrhosis. Full history taking and clinical examination were done. Patients with the following criteria were excluded: non-HCV-related liver cirrhosis as $\mathrm{HBV}$, autoimmune, being illiterate or having visual troubles, recent alcohol use, history or presence of overt hepatic encephalopathy, active infections, within 6 weeks gastrointestinal bleeding, renal impairment, electrolyte disturbances, recent use of psychotropic drugs or drugs improving encephalopathy as lactulose and rifaximin, transjugular intrahepatic portosystemic shunt, hepatocellular carcinoma, recent surgery, congestive heart failure, advanced pulmonary disease and psychiatric diseases.

All patients underwent abdominal ultrasonography, liver function tests, $\mathrm{CBC}$, INR, renal function tests and arterial ammonia measurement.

MHE was diagnosed using the critical flicker frequency [6], number connection test and serial dotting test [5].

\section{Calculations}

ALBI [7] $=\left(\log _{10}\right.$ bilirubin $\left.\mu \mathrm{mol} / \mathrm{L} \times 0.66\right)+($ albumin $\mathrm{g} /$ $\mathrm{L} \times-0.085)$.

ALBI grades: ALBI I $\leq-2.60$, ALBI II $>-2.60$ to $\leq$ -1.39 and ALBI III > -1.39 .

PALBI $[8]=\left(2.02 \times \log _{10}\right.$ bilirubin $)+\left(-0.37 \times\left[\log _{10}\right.\right.$ bilirubin $\left.]^{2}\right)+(-0.04 \times$ albumin $)+\left(-3.48 \times \log _{10}\right.$ platelets $)+\left(1.01 \times\left[\log _{10} \text { platelets }\right]^{2}\right)$.

ALBI-PLT [9] = sum of the ALBI grade (I-III) to the platelet count grade (I-II). Grade I platelet count = platelets $>150,000 / \mathrm{mm}^{3}$ and Grade II platelet count $=$ platelets $\leq 150,000 / \mathrm{mm}^{3}$. The ALBI PLT range is $2-5$.

ALBI score can be calculated online through the following link: https://www.mdcalc.com/albi-albuminbilirubin-grade-hepatocellular-carcinoma-hcc

\section{Statistical analysis}

Data were statistically analyzed using IBM $^{\ominus}$ SPSS $^{\oplus}$ Statistics $^{\bullet}$ version 21 for Windows (IBM Corporation, North Castle Drive, Armonk, New York, USA) and MedCalc ${ }^{\circ}$ version 18.2.1 (Seoul, Republic of Korea). Data were expressed as mean \pm standard deviation and row percentage for nominal data. All $p$ values are 2 tailed, with values $<0.05$ considered statistically significant.

Comparisons between two groups were performed using the Student's $t$ test for parametric data, and Mann-Whitney test for non-parametric data. CHIsquared test $\left(\chi^{2}\right)$ and Fisher exact test for categorical data analysis. The receiver operating characteristic (ROC) curve analysis was used for the detection of the cutoff value of the MHE presence. For each cutoff, sensitivity, specificity, positive predictive value and negative predictive value were calculated. The area under the curve (AUC) of different variables was compared using the DeLong tests to assess variable discrimination. Univariate and multivariate binary logistic regression were done for detecting the predictors of MHE.

\section{Results}

Our study included 257 patients that were diagnosed to have HCV-related liver cirrhosis. MHE was diagnosed in $64.6 \%$ of the patients and the rest $(35.4 \%)$ were free of MHE.

Patients with MHE compared with those without it (Table $1, p<0.05)$ were older $(54.61 \pm 8.3$ vs. $50.42 \pm$ 7.92 years) and were having higher values of serum total bilirubin $(1.14 \pm 0.73$ vs. $0.88 \pm 0.25 \mathrm{mg} / \mathrm{dL})$, INR $(1.34 \pm$ 0.28 vs. $1.18 \pm 0.14$ ), Child-Pugh (CTP) score (6.15 \pm 1.62 vs. $5.25 \pm 0.7)$ and MELD score $(10.78 \pm 4.01$ vs. $8.46 \pm 3.06)$. In addition, they had lower values of serum albumin $(3.46 \pm 0.52$ vs. $3.83 \pm 0.42 \mathrm{~g} / \mathrm{dL})$ and WBCs $\left(5.30 \pm 1.3\right.$ vs. $\left.5.70 \pm 1.36 \times 10^{3} / \mu \mathrm{L}\right)$. Patients with $\mathrm{MHE}$ were mainly CTP class B and C and also ALBI grade 2 and 3.

There was statistically significant difference (Table 1 and Figs. 1 and 2, $p<0.05$ ) between MHE patients and those MHE-free patients as regards ammonia (86.59 \pm 23.25 vs. $63.56 \pm 24.2)$, ALBI score $(-2.13 \pm 0.53$ vs. $-2.49 \pm 0.38)$, PALBI score $(-2.33 \pm 0.39$ vs. $-2.55 \pm$ $0.26)$ and ALBI-PLT (3.98 \pm 0.49 vs. $3.70 \pm 0.56)$. Both groups were the same for sex, serum AST, ALT, hemoglobin and platelets $(p>0.05)$.

By univariate logistic regression analysis, the following variables were associated with the existence of MHE as shown in Table $2(p<0.05)$; age (odds $=1.07$, 95\% C.I $=$ 1.03-1.1), total bilirubin (odds $=3.69,95 \%$ C.I $=1.65-$ 8.26 ), ammonia (odds $=1.05,95 \%$ C.I $=1.03-1.07$ ), CTP score $($ odds $=2.13,95 \%$ C.I $=1.48-3.07)$, MELD $($ odds $=$ 1.26, 95\% C.I $=1.09-1.44)$, ALBI (odds $=5.64$, 95\% C.I $=2.82-11.29$ ), PALBI (odds $=7.86$, 95\% C.I $=3.08-$ 20.09), ALBI-PLT (odds $=2.86$, 95\% C.I $=1.65-4.97$ ) but serum albumin was inversely related (odds $=2.86$, 95\% C.I $=0.10-0.37)$. On multivariate analysis, only age and ammonia was independently associated with MHE.

As shown in Table 3 and Fig. 3, ALBI cutoff $>-2.36$ had $57.23 \%$ sensitivity, $77.78 \%$ specificity, $84.1 \%$ PPV 
Table 1 Comparison of the baseline data of patient with and without MHE

\begin{tabular}{|c|c|c|c|c|}
\hline & \multirow{2}{*}{$\begin{array}{l}\text { None } \\
N=91(35.4 \%)\end{array}$} & MHE & \multirow[t]{2}{*}{$p$} \\
\hline & & & $N=166(64.6 \%)$ & \\
\hline \multicolumn{2}{|l|}{ Age (years) } & $50.42 \pm 7.92$ & $54.61 \pm 8.30$ & 0.001 \\
\hline \multirow[t]{2}{*}{ Sex } & Female & $46(50.5 \%)$ & $90(54.2 \%)$ & 0.603 \\
\hline & Male & 45 (49.5\%) & $76(45.8 \%)$ & \\
\hline \multicolumn{2}{|c|}{ Total bilirubin (mg/dL) } & $0.88 \pm 0.25$ & $1.14 \pm 0.73$ & $0.017 \#$ \\
\hline \multicolumn{2}{|c|}{ Direct bilirubin (mg/dL) } & $0.35 \pm 0.14$ & $0.53 \pm 0.48$ & $0.002 \#$ \\
\hline \multicolumn{2}{|c|}{ Albumin (g/dL) } & $3.83 \pm 0.42$ & $3.46 \pm 0.52$ & $0.001 \#$ \\
\hline \multicolumn{2}{|l|}{ AST (U/L) } & $42.54 \pm 22.03$ & $45.26 \pm 20.74$ & $0.061 \#$ \\
\hline \multicolumn{2}{|l|}{$\operatorname{ALT}(\mathrm{U} / \mathrm{L})$} & $34.80 \pm 20.12$ & $36.81 \pm 15.68$ & 0.391 \\
\hline \multicolumn{2}{|c|}{ Hemoglobin (g/dL) } & $11.97 \pm 1.48$ & $11.66 \pm 1.51$ & $0.160 \#$ \\
\hline \multicolumn{2}{|c|}{ WBCs $\left(\times 10^{3} / \mu \mathrm{L}\right)$} & $5.70 \pm 1.36$ & $5.30 \pm 1.30$ & 0.028 \\
\hline \multicolumn{2}{|c|}{ Platelets $\left(\times 10^{3} / \mu \mathrm{L}\right)$} & $122.37 \pm 40.49$ & $123.04 \pm 71.83$ & $0.515 \#$ \\
\hline \multicolumn{2}{|l|}{ INR } & $1.18 \pm 0.14$ & $1.34 \pm 0.28$ & $0.001 \#$ \\
\hline \multicolumn{2}{|c|}{ Ammonia $\mu \mathrm{mol} / \mathrm{L}$} & $63.56 \pm 24.20$ & $86.59 \pm 23.25$ & 0.001 \\
\hline \multicolumn{2}{|l|}{ CTP score } & $5.25 \pm 0.70$ & $6.15 \pm 1.62$ & 0.001\# \\
\hline \multirow[t]{3}{*}{ CTP class } & A & $86(43.2 \%)$ & $113(56.8 \%)$ & 0.001 \\
\hline & B & $5(10 \%)$ & 45 (90\%) & \\
\hline & C & $0(0 \%)$ & $8(100 \%)$ & \\
\hline \multicolumn{2}{|l|}{ MELD } & $8.46 \pm 3.06$ & $10.78 \pm 4.01$ & $0.001 \#$ \\
\hline \multicolumn{2}{|l|}{ ALBI score } & $-2.49 \pm 0.38$ & $-2.13 \pm 0.53$ & $0.001 \#$ \\
\hline \multirow[t]{3}{*}{ ALBI class } & ALBI 1 & $18(66.7 \%)$ & $9(33.3 \%)$ & 0.001 \\
\hline & ALBI 2 & 72 (34.1\%) & 139 (65.9\%) & \\
\hline & ALBI 3 & $1(5.3 \%)$ & $18(94.7 \%)$ & \\
\hline \multicolumn{2}{|l|}{ PALBI score } & $-2.55 \pm 0.26$ & $-2.33 \pm 0.39$ & 0.001 \\
\hline \multicolumn{2}{|c|}{ ALBI-PLT score } & $3.70 \pm 0.56$ & $3.98 \pm 0.49$ & $0.001 \#$ \\
\hline
\end{tabular}

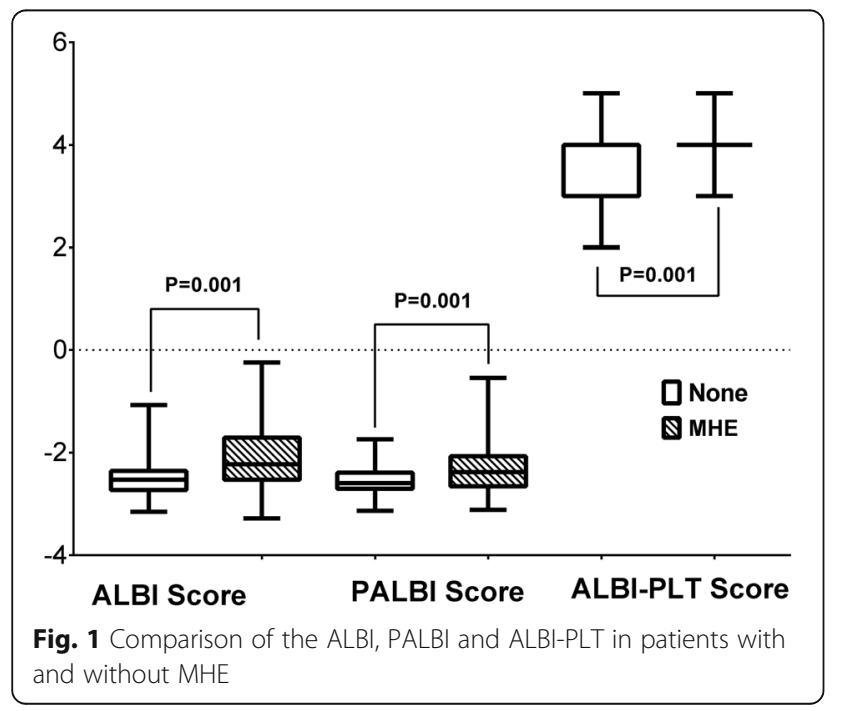

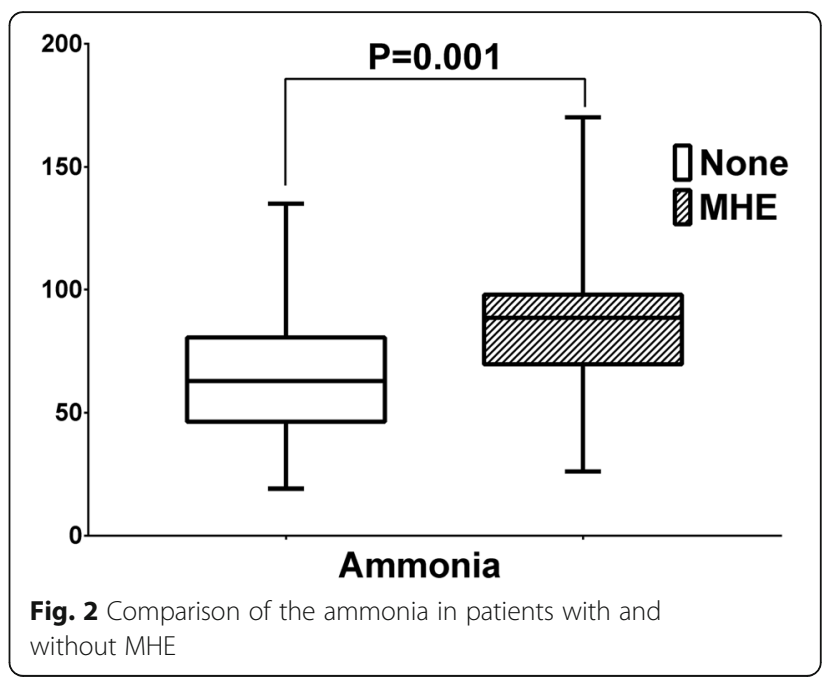

and $47 \%$ NPV $(p=0.001)$. PALBI cutoff $>-2.5$ had $60.84 \%$ sensitivity, $67.9 \%$ specificity, $79.5 \% \mathrm{PPV}$ and 45.8\% NPV $(p=0.001)$. ALBI-PLT cutoff $>3$ had $87.35 \%$ sensitivity, $27.16 \%$ specificity, $71.1 \%$ PPV and $51.2 \%$ NPV $(p=0.001)$. Ammonia cutoff $>76.5$ had 71.69\% sensitivity, $72.5 \%$ specificity, $91.5 \%$ PPV and $38.2 \%$ NPV $(p=0.001)$, CTP cutoff $>5$ had $45.8 \%$ sensitivity, 85.2\% specificity, 86.4\%PPV and 43.4\% NPV ( $p=$ $0.001)$ and MELD cutoff $>8.2$ had $65.9 \%$ sensitivity, 73.2\% specificity, $85.8 \%$ PPV and $39.7 \%$ NPV $(p=0.001)$.

On comparison of the AUC of the studied variables to detect the best one, ALBI is comparable to PALBI ( $p=$ $0.245)$ and ammonia $(p=0.603)$. PALBI is comparable to ammonia $(p=0.267)$. ALBI-PLT is inferior to ALBI $(p=0.018)$ and ammonia $(p=0.021)$ but comparable to PALBI $(p=0.281)$.

\section{Discussion}

MHE is a major health problem that is not under the spotlight because the patients look normal. It is catastrophic in many points. It prevents complex activities such as driving and planning a trip and impairs social

Table 2 Univariate and multivariate analysis of MHE predictors

\begin{tabular}{|c|c|c|c|c|c|c|}
\hline & \multicolumn{3}{|c|}{ Univariate } & \multicolumn{3}{|c|}{ Multivariate } \\
\hline & $p$ & Odds & 95\% C.I. & $p$ & Odds & 95\% C.I. \\
\hline Age & 0.001 & 1.07 & $1.03-1.10$ & 0.005 & 1.18 & $1.04-1.32$ \\
\hline Sex & 0.573 & 1.16 & $0.69-1.93$ & & & \\
\hline Ammonia & 0.001 & 1.05 & $1.03-1.07$ & 0.004 & 1.08 & $1.03-1.12$ \\
\hline CTP score & 0.001 & 2.13 & $1.48-3.07$ & 0.322 & 1.7 & $0.59-4.88$ \\
\hline MELD & 0.002 & 1.26 & $1.09-1.44$ & 0.200 & 1.26 & $0.88-1.79$ \\
\hline ALBI & 0.001 & 5.64 & $2.82-11.29$ & 0.438 & 10.85 & $0.02-4500.75$ \\
\hline PALBI & 0.001 & 7.86 & $3.08-20.09$ & 0.889 & 0.582 & $0.02-1480.90$ \\
\hline ALBI-PLT & 0.001 & 2.86 & $1.65-4.97$ & 0.555 & 0.45 & $0.03-6.48$ \\
\hline
\end{tabular}

CTP Child-Pugh, C.I Confidence interval 
Table 3 Receiver operating characteristic (ROC) curve analysis of ALBI, PALBI, ALBI-PLT and ammonia in patients with and without MHE

\begin{tabular}{|c|c|c|c|c|c|c|c|c|}
\hline & AUC & p & $95 \% \mathrm{Cl}$ & Cutoff & Sn & $\mathrm{Sp}$ & PPV & NPV \\
\hline CTP & 0.667 & 0.001 & $0.604-0.725$ & $>5$ & $45.8 \%$ & $85.2 \%$ & $86.4 \%$ & $43.4 \%$ \\
\hline MELD & 0.72 & 0.001 & $0.645-0.787$ & $>8.2$ & $65.9 \%$ & $73.2 \%$ & $85.8 \%$ & $39.7 \%$ \\
\hline ALBI & 0.739 & 0.001 & $0.649-0.766$ & $>-2.36$ & $57.23 \%$ & $77.78 \%$ & $84.1 \%$ & $47 \%$ \\
\hline PALBI & 0.705 & 0.001 & $0.597-0.719$ & $>-2.5$ & $60.84 \%$ & $67.9 \%$ & $79.5 \%$ & $45.8 \%$ \\
\hline ALBI-PLT & 0.650 & 0.001 & $0.545-0.67$ & $>3$ & $87.35 \%$ & $27.16 \%$ & $71.1 \%$ & $51.2 \%$ \\
\hline Ammonia & 0.768 & 0.001 & $0.705-0.824$ & $>76.5$ & $71.69 \%$ & $72.5 \%$ & $91.5 \%$ & $38.2 \%$ \\
\hline
\end{tabular}

AUC Area under the curve, C.I Confidence interval, Sn Sensitivity, Sp Specificity, PPV Positive predictive value, NPV Negative predictive value

interaction with poor health-related quality of life. Most patients have sleep troubles, short memory affection, work-related fatigue, poor driving, navigation skills. In addition, $40 \%$ of patients may develop falls, fractures with increased need for hospitalization and morbidity. MHE is an employment and socioeconomic burden since $60 \%$ of blue-collar workers are unfit to work compared with only $20 \%$ of white-collar workers [4].

MHE diagnosis needs sophisticated tests that may be copyrighted, need educated patients, training of the patients before doing the tests and may be costly [10]. There is an urgent need for a non-sophisticated diagnosis of MHE especially if using routine investigations.
Some biomarkers were useful in the identification of MHE as 3-nitro-tyrosine [11, 12], IL-6 and IL-18 [13], capillary blood ammonia bedside test following glutamine load [14] and venous ammonia [6].

MHE correlates with liver dysfunction so assessing the degree of the liver dysfunction may be an indirect method of MHE diagnosis or suspicion. CTP score is based on 5 variables but 2 of them are subjective.

ALBI, [7] PALBI [8] and ALBI-PLT [9] are models for assessment of the liver condition without subjective bias. They are based on routine simple investigations. They were studied mainly in patients with hepatocellular carcinoma and correlated with survival.

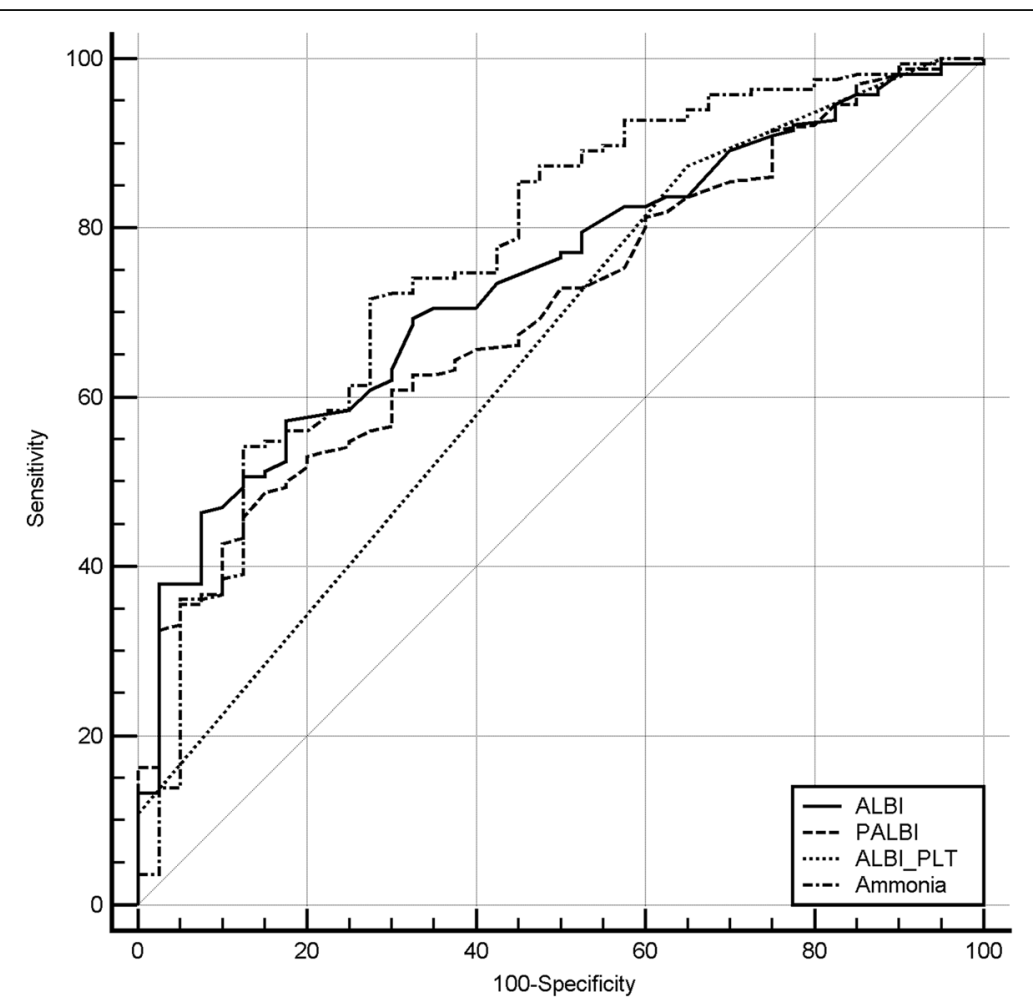

Fig. 3 The receiver operating characteristic (ROC) curve analysis of ALBI, PALBI, ALBI-PLT and ammonia in patients with and without MHE 
To our knowledge, this is the first study on assessing ALBI, PALBI and ALBI-PLT scores in patients with MHE.

In our study, most of the patients with MHE were mainly CTP B and C. Higher values of CTP, MELD, ALBI, PALBI, ALBI-PLT and ammonia levels were associated with MHE. Most patients were ALBI grade 2 and 3. The ALBI, PALBI, ALBI-PLT and ammonia had the following statistically significant cutoffs; >-2.36 (57.23\% sensitivity, $77.78 \%$ specificity), >-2.5 (60.84\% sensitivity, $67.9 \%$ specificity), > 3 (87.35\% sensitivity, $27.16 \%$ specificity) and $>76.5$ (69\% sensitivity, $72.5 \%$ specificity) respectively $(p=0.001)$. On comparison of the area under the curve to detect the best one; ALBI, PALBI and ammonia were comparable. ALBI-PLT was inferior to ALBI and ammonia but comparable to PALBI.

ALBI, PALBI, ALBI-PLT, ammonia, Child-Pugh scores were independent predictors of MHE. On multivariate analysis, only age and ammonia level were the only independent predictor of MHE.

Sharma and Sharma 2010 [6] conducted a study on 200 patients; only 82 (41\%) patients had MHE. MHE patients had statistically higher CTP score $(8.4 \pm 2.5$ vs. 7.7 $\pm 2.2)$, MELD (17.9 \pm 5.7 vs. $13.4 \pm 4.2)$ and ammonia $(104.8 \pm 37.9$ vs. $72.5 \pm 45.2 \mu \mathrm{mol} / \mathrm{L})$ that is in agreement with our study.

The incidence of MHE in their study was lower than reported in our study ( $41 \%$ vs. $64.6 \%$ ). The cutoff values in their study were higher than our cutoff values; CTP (7.5 vs. 5 ), MELD (15.5 vs. 8.2 ) and ammonia (84.5 vs. $76.5 \mu \mathrm{mol} / \mathrm{L}$ ). This may be ascribed to a small number of CTP C patients in our study.

\section{Limitation of the study}

small number of patients, small number of CTP class C patients, single-center experience and need to follow up the patients.

\section{Conclusion}

ALBI, PALBI and ammonia are clinical useful tools for the prediction of MHE.

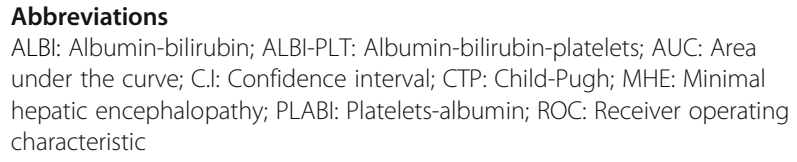
under the curve; C.I: Confidence interval; CTP: Child-Pugh; MHE: Minimal hepatic encephalopathy; PLABI: Platelets-albumin; ROC: Receiver operating characteristic

\section{Acknowledgements}

None

\section{Authors' contributions}

Data collection, study design and manuscript writing by AA. The author read and approved the final manuscript.

\section{Availability of data and materials}

The datasets used and/or analyzed during the current study available from the corresponding author on reasonable request.

Ethics approval and consent to participate

National Liver Institute IRB 00003415 . Written consent was obtained from the patients

\section{Consent for publication}

Not applicable.

\section{Competing interests}

The author delares that he/she has no competing interests.

Received: 28 September 2019 Accepted: 23 January 2020

Published online: 08 April 2020

\section{References}

1. Vilstrup H, Amodio P, Bajaj J, Cordoba J, Ferenci P, Mullen KD et al (2014) Hepatic encephalopathy in chronic liver disease: 2014 Practice Guideline by the American Association for the Study Of Liver Diseases and the European Association for the Study of the Liver. Hepatology 60:715-735

2. Weissenborn K (2015) Diagnosis of minimal hepatic encephalopathy. J Clin Exp Hepatol 5:S54-SS9

3. Flamm SL (2015) Covert hepatic encephalopathy. Clin Liver Dis 19:473-485

4. Agrawal S, Umapathy S, Dhiman RK (2015) Minimal hepatic encephalopathy impairs quality of life. J Clin Exp Hepatol 5:S42-SS8

5. Groeneweg M, Moerland W, Quero JC, Hop WC, Krabbe PF, Schalm SW (2000) Screening of subclinical hepatic encephalopathy. J Hepatol 32:748-753

6. Sharma P, Sharma B (2010) Predictors of minimal hepatic encephalopathy in patients with cirrhosis. Saudi J Gastroenterol 16:181-187

7. Johnson PJ, Berhane S, Kagebayashi C, Satomura S, Teng M, Reeves HL et al (2015) Assessment of liver function in patients with hepatocellular carcinoma: a new evidence-based approach-The ALBI Grade. J Clin Oncol 33:550-558

8. Elshaarawy O, Alkhatib A, Elhelbawy M, Gomaa A, Allam N, Alsebaey A, Rewisha E, Waked I (2019) Validation of modified albumin-bilirubin-TNM score as a prognostic model to evaluate patients with hepatocellular carcinoma. World J Hepatol 11(6):542-552

9. Chen P-H, Hsieh W-Y, Su C-W, Hou M-C, Wang Y-P, Hsin IF et al (2018) Combination of Albumin-bilirubin grade and platelet to predict compensated patient with hepatocellular carcinoma who do not require endoscopic screening for esophageal varices. Gastrointes Endo

10. Weissenborn K (2013) Psychometric tests for diagnosing minimal hepatic encephalopathy. Metabolic Brain Dis 28:227-229

11. Montoliu C, Cauli O, Urios A, ElMlili N, Serra MA, Giner-Duran R et al (2011) 3-Nitro-tyrosine as a peripheral biomarker of minimal hepatic encephalopathy in patients with liver cirrhosis. Am J Gastroenterol 106:1629

12. Felipo V, Urios A, Valero P, Sánchez M, Serra MA, Pareja I et al (2013) Serum nitrotyrosine and psychometric tests as indicators of impaired fitness to drive in cirrhotic patients with minimal hepatic encephalopathy. Liver Int 33 1478-1489

13. Montoliu C, Piedrafita B, Serra MA, del Olmo JA, Urios A, Rodrigo JM et al (2009) IL-6 and IL-18 in Blood may discriminate cirrhotic patients with and without minimal hepatic encephalopathy. J Clin Gastroenterol 43:272-279

14. Ditisheim S, Giostra E, Burkhard PR, Goossens N, Mentha G, Hadengue A et al (2011) A capillary blood ammonia bedside test following glutamine load to improve the diagnosis of hepatic encephalopathy in cirrhosis. BMC Gastroenterol 11:134

\section{Publisher's Note}

Springer Nature remains neutral with regard to jurisdictional claims in published maps and institutional affiliations. 